Letter to the Editor

\title{
The percutaneous closure of patent foramen ovale (PFO): Impact on the quality of life
}

\author{
Salvatore Evola, B.A. Waseem Kauroo*, Rosaria Linda Trovato, Luigi Alioto, Giovanni D'Amico, \\ Giuseppe Fonte, Giuseppe Andolina, Salvatore Novo, Pasquale Assennato
}

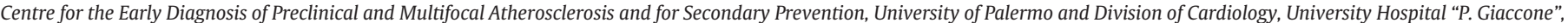
Department of Internal Medicine and Cardiovascular Disease, Palermo, Italy

\section{A R T I C L E I N F O}

Article history:

Received 5 December 2012

Accepted 18 January 2013

Available online $\mathrm{xxxx}$

\section{Keywords:}

Patent foramen ovale

Cryptogenic stroke

Migraine with aura

Patent foramen ovale (PFO) is an abnormal communication between the right and the left atrium due to an incomplete fusion between septum primum and septum secundum [1]. It involves $25-30 \%$ of the general population [2]. PFO is often associated to cryptogenic cerebral vascular accident (CVA) [3,4] and migraine with aura [5]. PFO can be also associated with other anatomical abnormalities (Chiari network, septal aneurysm, eustachian valve) which can increase the shunt [1].

As the prevailing increase of PFO percutaneous interventions, we found interesting to assess the "Quality of Life" after percutaneous closure, intending to describe the state of physical, psychological and social well-being.

In our center between July 2009 and July 2012, the percutaneous closure of PFO was performed in 34 patients: 20 women $(58.8 \%)$ and 14 men (41.2\%); mean age $46 \pm 9.67$. The diagnosis of right to left shunt was achieved by the bubble test ( $9 \mathrm{ml}$ of saline and $1 \mathrm{ml}$ of air) with the transcranial Doppler (TCDc). Then a trans-thoracic echocardiography (TTE) confirmed the right to left shunt of micro-bubbles and allowed to find out the presence of atrial septal aneurysm (ASA) and other anatomical anomalies such as the Chiari network and the eustachian valve. The anatomical and functional characteristics of PFO were then better studied with transesophageal echocardiography (TEE)-(Fig. 1).

The indications employed for percutaneous treatment, after careful echocardiographic studies and neurological consults, consist in the occurrence of ischemic events TIA/STROKE, classified as cryptogenic nature ( $65 \%$ of our treated patients), often recurrent, or the presence of multiple cerebral ischemic lesions detected with MRI, even if asymptomatic, for

* Corresponding author at: Vicolo Paterna n.17, 90133 Palermo Sicily/Italy. Tel.: + 39 $091583627,+393297825915$.

E-mail address: waseemkauroo@hotmail.com (B.A.W. Kauroo). which no valid etiopathogenetic cause could be identified. Moreover $56 \%$ complained frequent and debilitating episodes of migraine with aura before treatment.

Percutaneous PFO closure was performed under local anesthesia with intracardiac ultrasound guidance (9 MHz Ultra ICE, Boston Scientific Corporation, San Jose, California) to optimize the positioning of the devices. The PFO devices used up to now are: Amplatzer ${ }^{\circledR}(4$ patients $11.8 \%$ ), Cardia ${ }^{\circledR}$ (4 patients $11.8 \%$ ), and Figulla ${ }^{\circledR}$ Flex PFO Occluder (26 patients $76.4 \%$ ). All patients were then treated with acetylsalicylic acid $100-325 \mathrm{mg} /$ die and Ticlopidine $500 \mathrm{mg} / \mathrm{die}$ or Clopidogrel $75 \mathrm{mg} / \mathrm{die}$ for the first six months and then only acetylsalicylic acid for another 6 months.

The follow-up includes a Holter-ECG (7 days after closure) and then programmed controls: TTE at 1 and 3 months, TTE + TCDc at 6 months and at 1 year.

After informed consent, our treated patients were subjected to a validated questionnaire, "Quality of Life" (QoL) SF 36 V1 standard [6], administered before PFO closure and then after 6 months. The questionnaire is a self-compile generic test on quality of life studying multiple aspects of the psycho-physical well-being of the subject through 36 questions. The result obtained from the responses is represented by 8 scales, which is a quantification of a specific aspect of health status.

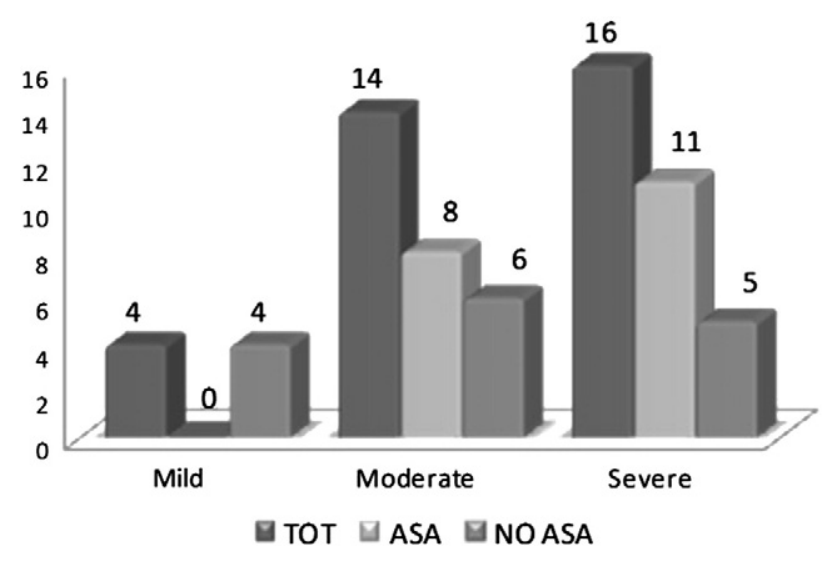

Fig. 1. Echocardiography ultrasound characteristics. 
Table 1

Pre/post treatment mean values and standard deviations.

\begin{tabular}{|c|c|c|c|c|c|}
\hline & Mean pre-treatment & Mean post-treatment & Standard dev. pre-treatment & Standard dev. post treatment & Paired probability \\
\hline Physical activity & 66.72 & 85.34 & 28.32 & 19.31 & $\mathrm{p}=0.001$ \\
\hline Physical role limitation & 42.24 & 81.89 & 43.35 & 29.04 & $\mathrm{p}=0.0001$ \\
\hline Migraine & 59.45 & 80.55 & 27.82 & 24.47 & $\mathrm{p}=0.0001$ \\
\hline General health & 48.93 & 71.62 & 22.58 & 24.3 & $\mathrm{p}<0.0001$ \\
\hline Vitality & 52.93 & 73.2 & 23.99 & 21.7 & $\mathrm{p}<0.0001$ \\
\hline Social activities & 60.82 & 82.24 & 27.65 & 21.57 & $\mathrm{p}=0.0001$ \\
\hline Emotional role limitation & 49.27 & 82.24 & 42.37 & 22.5 & $\mathrm{p}<0.0001$ \\
\hline Mental health & 56.27 & 82.24 & 22.24 & 17.85 & $\mathrm{p}<0.0001$ \\
\hline
\end{tabular}

Among our 34 treated patients, 4 were excluded from the study for recent closure; 29 patients were subjected to QoL questionnaire while $\mathrm{n}=1$ was unavailable.

The mean values obtained on different fields, from the analysis of the questionnaires, before and after treatment administration, were compared using the Student $t$-test for paired samples, in order to assess the statistical significance of improvements on quality of life. The obtained values are expressed as mean $\pm 1 \mathrm{SD}$. A p value of $<0.05$ was considered statistically significant.

The procedure was successfully performed in 34 treated patients. No complications were recorded during the procedure. The presence of new-onset atrial fibrillation (AF) was excluded. A closure rate of 86.6\% was obtained and no cerebrovascular symptomatic events occurred in treated patients, until now. The QoL SF-36 questionnaire reported a positive impact of the procedure on the quality of life by re-appropriation of normal daily activities; also from the psychological point of view. The comparison of the mean values obtained from the analysis on different fields of the questionnaire, before and after treatment, has suggested a statistically significant improvement in quality of life (Table 1).

This result is reasonably related to the improvement of migraine patients which were confined below all aspects. Among our 19 patients which were affected from severe migraine with aura, 94.7\% had in fact a significant benefit in health, due to an important reduction of the migraine frequency and severity or even their interruption $(\mathrm{p}=0.0001)$.

In patients with cryptogenic stroke and PFO, the percutaneous closure treatment represents a possible and effective procedure and a possible alternative to the only antiplatelet or anticoagulant medical therapy conducted for a lifetime [7,8], even if the first randomized controlled trial, CLOSURE I,has not demonstrated the superiority of the percutaneous treatment against the medical therapy [9]. In our center the percutaneous technique was initiated relatively recently, however our results are encouraging. We considered only the presence of CVA as the main indication for percutaneous treatment of PFO while patients with migraine with aura were treated only with a medical therapy, according to the 2010 Italian SPREAD (Stroke Prevention and Educational Awareness Diffusion) guidelines (VI edition).

Although migraine with aura does not represent an indication for percutaneous closure, and the results of the MIST I [10] were definite- ly lower than expected, the finding of a clinical improvement of this disease, bodes well for a substantial improvement in quality of life for these patients. Also the psychological aspect should not be underestimated. The prospect of an effective secondary prevention of ischemic recurrences, through a permanent treatment, could certainly contribute to improve the quality of life and interpersonal relationships of these subjects.

The percutaneous closure of the PFO seems to lead a good impact on the quality of life compared to the life condition prior the closure treatment. Certainly, further studies with larger cases are necessary to assess the real benefit of PFO percutaneous closure. Therefore we suggest to use common sense in the choice and selection of patients for the indication of percutaneous closure, and particularly to take in more consideration also those with the only migraine with aura.

\section{References}

[1] Calvert Patrick A, Rana Bushra S, Kydd Anna C, et al. Patent foramen ovale: anatomy, outcomes, and closure. Nat Rev Cardiol 2011 Mar;8(3):148-60.

[2] Meissner I, Khandheria BK, Heit JA, et al. Patent foramen ovale: innocent or guilty? Evidence from a perspective population-based study. J Am Coll Cardiol Jan. 17 2006;47(2):440-5.

[3] Homma S, Di Tullio MR. Patent foramen ovale and stroke. J Cardiol 2010 Sep;56(2): 134-41.

[4] Fazio G, Ferro G, Barbaro G, Ferrara F, Novo G, Novo S. Patent foramen ovale and thromboembolic complications. Curr Pharm Des 2010;16(31):3497-502.

[5] Wilmshurst P, Nightingale S. The role of cardiac and pulmonary pathology in migraine: a hypothesis. Headache Mar. 2006;46(3):429-34.

[6] Anderson C, Laubscher S, Burns R. Validation of the short form 36 (SF-36) health survey questionnaire among stroke patients. Stroke 1996 Oct;27(10):1812-6.

[7] Windecker S, Wahl A, Nedeltchev K, et al. Comparison of medical treatment with percutaneous closure of patent foramen ovale in patients with cryptogenic ictus. J Am Coll Cardiol 2004;44:750-8.

[8] Heinisch C, Bertog S, Wunderlich N, et al. Percutaneous closure of the patent foramen ovale using the HELEX ${ }^{\circledR}$ Septal Occluder: acute and long-term results in 405 patients. Eurolntervention 2012 Oct 22;8(6):717-23.

[9] Furlan AJ, et al. Study design of the CLOSURE I trial. A prospective, multicenter, randomized, controlled trial to evaluate the safety and efficacy of the Starflex septal closure system versus best medical therapy in patients with stroke or transient ischemic attack due to presumed paradoxical embolism through a patent foramen ovale. Stroke Dec. 2010;41(12):2872-83.

[10] Migraine Intervention with STARflex Technology (MIST) Trial. A prospective, multicenter, double-blind, sham-controlled trial to evaluate the effectiveness of patent foramen ovale closure with STARflex septal repair implant to resolve refractory migraine headache. Circulation Mar. 18 2008;117(11):1397-404. 